Pacific Journal of Mathematics

SAMPLE FUNCTION BEHAVIOR OF INCREASIN
PROCESSES WITH STATIONARY, INDEPENDENt
INCREMENt

BERT E. FRISTEDT 


\title{
SAMPLE FUNCTION BEHAVIOR OF INCREASING PROCESSES WITH STATIONARY, INDEPENDENT INCREMENTS
}

\author{
Bert E. FRISTEdT
}

In this paper we are concerned with the sample functions of increasing stochastic processes, $X_{\nu}$, having stationary, independent increments; normalized so that $X_{\nu}$ has no deterministic linear component and $X_{\nu}(0)=0$, (i.e., $X_{\nu}$ is a subordinator).

For $h$ a fixed function, we are interested in the following two events:

$$
\begin{aligned}
& \left\{\omega: X_{\nu}(t, \omega)>h(t) \text { infinitely of ten as } t \rightarrow 0\right\}, \\
& \left\{\omega: X_{\nu}(t, \omega)>h(t) \text { infinitely of ten as } t \rightarrow \infty\right\} .
\end{aligned}
$$

In case $X_{\nu}$ is a stable process, Khinchin has given integral tests to apply to a wide class of $h^{\prime} s$ in order to decide whether one, the other, or both of these two events have probability zero or one. The purpose of this paper is to give similar results, without assuming $X_{\nu}$ to be stable.

We also prove (Theorem 3) a variation-type theorem concerning the sample functions. Theorem 4 is an $L_{1}$ convergence theorem for the distribution function as time goes to zero.

2. Notation. We let $\nu$ be a measure on $(0, \infty)$ with

$$
\int_{0}^{\infty} y(1+y)^{-1} \nu(d y)<\infty .
$$

Then, we let

$$
\begin{aligned}
& g_{\nu}(u)=\int_{0}^{\infty}\left(1-e^{-u y}\right) \nu(d y) ; \\
& \varphi_{\nu}(t, u)=\exp \left(-t g_{\nu}(u)\right) .
\end{aligned}
$$

We let $X_{\nu}$ be a function of two variables; the first variable being an element in $[0, \infty)$ and the second variable being an element in a probability space $\Omega$ with probability measure $P$. We take $X_{\nu}(0, \omega)=0$ for all $\omega \in \Omega$, and we take $X_{\nu}(\cdot, \omega)$ to be an increasing right continuous function (The range of $X_{\nu}$ is taken to be a subset of $[0, \infty)$.). We require that

$$
\int_{0}^{\infty} e^{-u x} d_{2} F_{\nu}(t, x)=\varphi_{\nu}(t, u)
$$

where 


$$
F_{\nu}(t, x)=P\left\{\omega: X_{\nu}(t, \omega)<x\right\} .
$$

It follows ([1] and [6], 417-424) that $X_{\nu}$ is an increasing stochastic process having stationary, independent increments. Moreover, if $Y(t, \omega)=X_{\nu}(t, \omega)-c t$ where $c>0$, then $Y$ will not be an increasing process (i.e. $X_{\nu}$ has no deterministic linear component.). It is true ([1] and [6], 417-424) that every increasing stochastic process having stationary, independent increments and having the property mentioned in the preceding sentence-whose value at 0 is 0 and which is right continuous-is an $X_{\nu}$ for an appropriate choice of $\nu$. The measure $\nu$ is called the Levy measure corresponding to the process $X_{\nu}$.

If we remove an appropriate set of measure zero from $\Omega$, we can make some further interesting statements ([4], 513, and [6], 417-424). Let $J_{\nu}(t, \omega)=X_{\nu}(t, \omega)-X_{\nu}(t-, \omega)$. Except for a countable number of $t$ (depending on $\omega), J_{\nu}(t, \omega)=0$. In addition,

$$
X_{\nu}(t, \omega)=\sum_{\tau \leqq t} J_{\nu}(\tau, \omega) .
$$

If $A \subset[0, \infty) x(0, \infty)$ and if $A$ is measurable, we let $N_{\nu}(A, \omega)$ equal the number of $t$ for which $\left(t, J_{\nu}(t, \omega)\right) \in A$. Then, the random variable, $N_{\nu}(A, \cdot)$, has a Poisson distribution with parameter $(\lambda \times \nu)(A)$ where $\lambda$ is Lebesque measure. [If $(\lambda \times \nu)(A)=\infty$, then $P\left\{\omega: N_{\nu}(A, \omega)=\infty\right\}=1$.] If $A, B \subset[0, \infty) \times(0, \infty), A$ and $B$ are measurable and $A \cap B=\varphi$, then $N_{\nu}(A, \cdot)$ and $N_{\nu}(B, \cdot)$ are independent random variables.

Three standard types of abbreviations will often be used: they are illustrated by the following "equalities:"

(i) $X_{\nu}(t)=X_{\nu}(t, \omega)=X_{\nu}(t, \cdot)$;

(ii) $P\left\{X_{\nu}(t)<x\right\}=P\left\{\omega: X_{\nu}(t, \omega)<x\right\}$;

(iii) $\left\{\omega: X_{\nu}(t, \omega) \geqq h(t)\right.$ i.o. as $\left.t \rightarrow 0\right\}$

$=\left\{X_{\nu}(t) \geqq h(t)\right.$ i.o. as $\left.t \rightarrow 0\right\}$

$=\{\omega$ : Given $T>0, \exists t(T, \omega) \ni 0<t(T, \omega)<T$

and $X_{\nu}(t(T, \omega), \omega) \geqq h(t(T, \omega))$. $\}$.

Our final item of notation is as follows:

$M=\{h: h$ is a strictly increasing function from $[0, \infty)$ onto $[0, \infty)$ and $h$ is concave upward $\}$.

Note. $h$ is concave upward means that

$$
h(\lambda a+(1-\lambda) b) \leqq \lambda h(a)+(1-\lambda) h(b), \quad 0<\lambda<1 .
$$

If $h \in M$, we write $X_{\nu \circ h}(t, \omega)=\sum_{\tau \leqq t} h^{-1}\left(J_{\nu}(\tau, \omega)\right)$.

3. Theorems and proofs. In a first reading of Theorems 1 and 2 , the reader is advised to skip over statements labeled with a primed numeral. 
THeOREM 1. Let $h \in M$. Then the following statements are equivalent:

(i) $P\left\{X_{\nu}(t) \geqq h(t)\right.$ i.o. as $\left.t \rightarrow 0\right\}=0$;

( i' ) $P\left\{X_{\nu}(t)>h(t)\right.$ i.o. as $\left.t \rightarrow 0\right\}=0$;

(ii) $\int_{0}^{1}\left[1-F_{\nu}(t, h(t))\right] / t d t<\infty$;

(ii') $\int_{0}^{1}\left[1-F_{\nu}(t, h(t)+)\right] / t d t<\infty ;$

(iii) $\int_{0}^{1-} h^{-1}(y) \nu(d y)<\infty$;

(iii') $\int_{0}^{1} \nu[h(t), \infty) d t=\int_{0}^{1} \nu(h(t), \infty) d t<\infty ;$

(iv) $\int_{0}^{1}\left[g_{\nu}\left(\frac{1}{h(t)}\right)-\frac{1}{h(t)} g_{\nu}^{\prime}\left(\frac{1}{h(t)}\right)\right] d t<\infty$.

If statements (i) and ( $\left.i^{\prime}\right)$ are false, then true statements are obtained by replacing 0 by 1 in the right hand sides of these statements. If $h_{c}(t)=h(c t)$ and $H_{c}(t)=c h(t), 0<c<\infty$, then $h_{c} \in M$ and $H_{c} \in M$, and the truth or falsity of the above statements is not changed by replacing $h$ by $h_{c}$ or $H_{c}$. The integrals in (ii) and (ii') conceivably might not exist (even in the sense of $=\infty$ ): if so, either upper or lower integrals may be used.

REMARK. If $h(t)=t$, then $h \in M$ and statement (iii) is true. On the other hand, if $\nu(0, \infty)=\infty$, then there exists an $h \in M$ such that statement (iii) is false. If $\nu(0, \infty)<\infty$, then $P\left\{X_{\nu}(t)>0\right.$ i.o. as $t \rightarrow 0\}=0$.

REMARK. Theorem 3 of [9] together with $h(t)=t^{1 / 2}$ shows us that statements (i) and (iii) are not equivalent if we do not assume $X_{\nu}$ to be increasing or $h \in M$.

REMARK. The term $\left[-\frac{1}{h(t)} g_{\nu}^{\prime}\left(\frac{1}{h(t)}\right)\right]$ cannot be dropped from proposition (iv). One obtains a counter-example by taking $h(t)=t$ and

$$
v[y, \infty)= \begin{cases}y^{-1}(\log y)^{-2}, & y \leqq .1 \\ 0 \quad, & y>.1\end{cases}
$$

Proof. Part 0. We shall assume that the integrals in parts (ii) and (ii') exist (We do not exclude $=\infty$.): if not, only minor changes are needed in the proof.

Part 1. Obviously (i) $\Rightarrow$ (i') and (ii) $\Rightarrow$ (ii').

Part 2. We prove (iii) $\Leftrightarrow\left(\right.$ iii' $\left.^{\prime}\right)$. Using integration by parts, we obtain 


$$
h^{-1}(1) \nu[1, \infty)+\int_{\delta+}^{1-} h^{-1}(y) \nu(d y)=h^{-1}(\delta) \nu(\delta, \infty)+\int_{\delta}^{1} \nu(y, \infty) d h^{-1}(y) .
$$

We note that

$$
h^{-1}(\delta) \nu(\delta, \infty) \leqq \int_{0}^{\delta} \nu(y, \infty) d h^{-1}(y)
$$

It follows that (iii) $\Leftrightarrow\left(\right.$ (iii') $^{\prime}$ once it is realized that the statement

$$
\int_{0}^{1} \nu(y, \infty) d h^{-1}(y)<\infty
$$

is equivalent to (iii').

Part 3. We prove $\left(\mathrm{i}^{\prime}\right) \Rightarrow\left(\right.$ iii' $\left.^{\prime}\right)$. Let $A=\{(t, x): t \in[0,1], x>h(t)\}$. Then, since $X_{\nu}(t, \omega) \geqq J_{\nu}(t, \omega), N_{\nu}(A)$ is finite with probability one if $\left(i^{\prime}\right)$ is true; and hence, if $\left(i^{\prime}\right)$ is true, $(\lambda \times \nu)(A)<\infty$. But

$$
(\lambda \times \nu)(A)=\int_{0}^{1} \nu(h(t), \infty) d t .
$$

Part 4. We now prove (iii) $\Rightarrow$ (i). Since $h^{-1}$ is concave downward, we have

$$
X_{\nu \circ h}(t, \omega)=\sum_{\tau \leqq t} h^{-1}\left(J_{\nu}(\tau, \omega)\right) \geqq h^{-1}\left(\sum_{\tau \leqq t} J_{\nu}(\tau, \omega)\right)=h^{-1}\left(X_{\nu}(t, \omega)\right) .
$$

So we can prove (i) by proving

$$
P\left\{X_{\nu \circ h}(t) \geqq t \text { i.o. as } t \rightarrow 0\right\}=0 \text {. }
$$

The notation $X_{\nu \circ h}$ is appropriate since it is easy to see that $X_{\nu \circ h}$ is the increasing process corresponding to the Levy measure given by

$$
\mu[y, \infty)=(\nu \circ h)[y, \infty)=\nu[h(y), \infty) \text { (i.e. Notation: } \mu=\nu \circ h) .
$$

The only thing to be checked is that

$$
\int_{0}^{\infty} y(1+y)^{-1} \mu(d y)<\infty .
$$

But this statement is clearly equivalent to (iii).

If $P\left\{X_{\mu}(t) \geqq t\right.$ i.o. as $\left.t \rightarrow 0\right\} \neq 0$, then, by Blumenthal's $0-1$ law (page 57, [3]), $P\left\{X_{\mu}(t) \geqq t\right.$ i.o. as $\left.t \rightarrow 0\right\}=1$. We consider the set $\left\{\left(t_{0}, \omega\right): t_{0} \in[0,1), X_{\mu}\left(t_{0}+t, \omega\right)-X_{\mu}\left(t_{0}, \omega\right) \geqq t\right.$ i.o. as $\left.t \downarrow 0\right\}$. This set is measurable since it equals

$$
\begin{gathered}
\bigcap_{n=1}^{\infty} \bigcup_{m=n}^{\infty} \bigcap_{k=1}^{\infty} \bigcup_{r} \bigcup_{2^{-m} \leqq s \varliminf^{-m+1}}\left\{\left(t_{0}, \omega\right): 0 \leqq t_{0}, r-2^{-k} \leqq t_{0} \leqq r,\right. \\
\left.X(r+s, \omega)-X(r, \omega) \geqq s-2^{-k}\right\}
\end{gathered}
$$

where $r$ and $s$ are rational. Thus, by Fubini's Theorem it follows that 


$$
\begin{aligned}
& P\left\{\text { Lebesgue measure of } \left\{t_{0}: t_{0} \in[0,1)\right.\right. \text { and } \\
& \left.\left.X_{\mu}\left(t_{0}+t\right)-X_{\mu}\left(t_{0}\right) \geqq t \text { i.o. as } t \downarrow 0\right\}=1\right\}=1 .
\end{aligned}
$$

For each $\omega$ consider all intervals $\left[t_{1}, t_{2}\right] \subset[0,1)$ having the property that

$$
X_{\mu}\left(t_{2}, \omega\right)-X_{\mu}\left(t_{1}, \omega\right) \geqq t_{2}-t_{1} .
$$

For a subset, $\Omega^{\prime}$, of $\Omega$ having probability one, the set of all such intervals covers, in the sense of Vitali, a subset of $[0,1)$ having Lebesque measure equal to one. If $\varepsilon>0$ and $\omega \in \Omega^{\prime}$, we conclude, by the Vitali covering theorem that there exists a sequence

$$
0 \leqq t_{1}(\omega)<t_{2}(\omega)<\cdots<t_{2 n}(\omega) \leqq 1
$$

such that

$$
\begin{aligned}
& X_{\mu}\left(t_{2 k}(\omega), \omega\right)-X_{\mu}\left(t_{2 k-1}(\omega), \omega\right) \geqq t_{2 k}(\omega)-t_{2 k-1}(\omega), k=1, \cdots, n ; \\
& \sum_{k=1}^{n}\left[t_{2 k}(\omega)-t_{2 k-1}(\omega)\right]>1-\varepsilon .
\end{aligned}
$$

Hence, for $\omega \in \Omega^{\prime}, X_{\mu}(1, \omega) \geqq 1$.

Thus, we have arrived at a contradiction since $F_{\mu}(1,1)>0$ which is a consequence of the fact that $X_{\mu}$ has no deterministic linear component; in fact, $F_{\mu}(t, x)>0$ if $x>0$. This might be a good point to mention that it has been proved in [1] that the formulas (given at the beginning of $\S 2$ of this paper) characterizing $X_{\mu}$ guarantee that $X_{\mu}$ has no negative deterministic linear component; but I have not seen in the literature any explicit proof of the fact that these formulas guarantee that $X_{\mu}$ has no positive deterministic linear component. Let us look at a proof of this fact.

If $F_{\mu}(t, x)=0$ and $x>0$, then

$$
\begin{aligned}
1 & -\exp \left(-t \int_{0}^{\infty}\left(1-e^{-u z}\right) \mu(d z)\right) \\
& =\int_{0}^{\infty}\left(1-e^{-u y}\right) d_{2} F_{\mu}(t, y) \\
& =\int_{x}^{\infty}\left(1-e^{-u y}\right) d_{2} F_{\mu}(t, y) \geqq 1-e^{-u x} .
\end{aligned}
$$

Hence,

$$
\int_{0}^{\infty}\left(1-e^{-u z}\right) \mu(d z) \geqq u x / t
$$

But

$$
\begin{aligned}
& u^{-1} \int_{0}^{\infty}\left(1-e^{-u z}\right) \mu(d z) \\
& \quad \leqq \int_{0}^{(1 / u)+} z \mu(d z)+u^{-1} \mu\left(u^{-1}, \infty\right) \rightarrow 0 \text { as } u \rightarrow \infty .
\end{aligned}
$$


Thus, we have arrived at the desired contradiction.

I wish to thank Professor Steven Orey for some helpful suggestions on simplifying this part of the proof: my original proof was much more computational in nature.

Part 5. Proposition (iii') is true if and only if it is true with $h$ replaced by $h_{c}$ : this follows by a simple change of variables. Since $h$ is concave upwards, we have

$$
\begin{array}{ll}
h(t) \leqq H_{c}(t) \leqq h_{c}(t), & c \geqq 1 ; \\
h(t) \geqq H_{c}(t) \geqq h_{c}(t), & c \leqq 1 .
\end{array}
$$

Hence, proposition (iii') is true if and only if it is true with $h$ replaced by $H_{c}$. Moreover, since we have now proved statements (i), (i'), and (iii) to be equivalent to (iii'), we can make similar assertions about (i), (i'), and (iii).

Part 6. We now prove (ii') $\Rightarrow$ (i). Now (ii) implies

$$
\begin{aligned}
\infty & >\int_{0}^{1}\left[1-F_{\nu}\left(t, h_{5}(t / 4)\right)\right] / t d t=\sum_{n=0}^{\infty} \int_{2^{-n-1}}^{2_{-n}^{-n}}\left[1-F_{\nu}\left(t, h_{5}(t / 4)\right)\right] / t d t \\
& \geqq \sum_{n=0}^{\infty}\left(2^{-n}-2^{-n-1}\right) 2^{n}\left[1-F_{\nu}\left(2^{-n-1}, h_{5}\left(2^{-n-2}\right)\right)\right] \\
& =\frac{1}{2} \sum_{n=0}^{\infty}\left[1-F_{\nu}\left(2^{-n-1}, h_{5}\left(2^{-n-2}\right)\right)\right] .
\end{aligned}
$$

Hence, only finitely many of the following events can occur:

$$
\left\{X_{\nu}\left(2^{-n-1}\right) \geqq h_{5}\left(2^{-n-2}\right)\right\} ; \quad n=0,1,2, \cdots
$$

Thus certainly only finitely many of the following events can occur:

$$
\left\{X_{\nu}(t) \geqq h_{5}(t) \text { for some } t \in\left[2^{-n-2}, 2^{-n-1}\right]\right\} ; \quad n=0,1,2, \cdots
$$

Therefore, using part 5 also, we conclude that (i) is true.

Part 7. We assume (i) to be true and prove (ii). By part 5, we assume (i) to be true with $h$ replaced by $h_{1 / 4}$. Hence, with probability 1 , only finitely many of the following mutually independent events can occur:

$$
\left\{X_{\nu}\left(2^{-n}\right)-X_{\nu}\left(2^{-n-1}\right) \geqq h_{1 / 4}\left(2^{-n}\right)\right\}, \quad n=0,1,2, \cdots ;
$$

or equivalently, the events

$$
\left\{X_{\nu}\left(2^{-n}\right)-X_{\nu}\left(2^{-n-1}\right) \geqq h\left(2^{-n-2}\right)\right\}, \quad n=0,1,2, \cdots
$$

Therefore,

$$
\begin{aligned}
\infty & >\sum_{n=0}^{\infty}\left[1-F_{\nu}\left(2^{-n-1}, h\left(2^{-n-2}\right)\right)\right] \\
& \geqq \sum_{n=0}^{\infty} \int_{\Sigma^{-n-2}}^{2^{-n-1}}\left[1-F_{\nu}(t, h(t))\right] / t d t=\int_{0}^{1 / 2}\left[1-F_{\nu}(t, h(t))\right] / t d t .
\end{aligned}
$$


Part 8. Assume that (iv) is true. Then

$$
\begin{aligned}
\infty & >\int_{0}^{1} \int_{0}^{\infty}\left[1-\left(1+\frac{y}{h(t)}\right) \exp \left(-\frac{y}{h(t)}\right)\right] \nu(d y) d t \\
& \geqq \int_{0}^{1} \int_{h(t)+}^{\infty}\left[1-\left(1+\frac{y}{h(t)}\right) \exp \left(-\frac{y}{h(t)}\right)\right] \nu(d y) d t \\
& \geqq\left(1-2 e^{-1}\right) \int_{0}^{1} \nu(h(t), \infty) d t .
\end{aligned}
$$

At the last step we used the fact that $(1+a) e^{-a}$ decreases as a increases. We have proved that (iv) $\Rightarrow$ (iii').

Part 9. We assume that (iii) and (iii') are true and prove (iv). We note that $1-(1+a) e^{-a} \leqq a^{2} / 2$ if $a \geqq 0$. Then,

$$
\begin{aligned}
\int_{0}^{1} & {\left[g_{\nu}\left(\frac{1}{h(t)}\right)-\frac{1}{h(t)} g_{\nu}^{\prime}\left(\frac{1}{h(t)}\right)\right] d t } \\
= & \int_{0}^{1} \int_{0}^{\infty}\left[1-\left(1+\frac{y}{h(t)}\right) \exp \left(-\frac{y}{h(t)}\right)\right] \nu(d y) d t \\
= & \int_{0}^{1} \int_{0}^{h(t)+}\left[1-\left(1+\frac{y}{h(t)}\right) \exp \left(-\frac{y}{h(t)}\right)\right] \nu(d y) d t \\
& +\int_{0}^{1} \int_{h(t)+}^{\infty}\left[1-\left(1+\frac{y}{h(t)}\right) \exp \left(-\frac{y}{h(t)}\right)\right] \nu(d y) d t \\
& \leqq
\end{aligned}
$$

The second term is finite. Since $h$ is concave upward, the first term is no larger than

$$
\begin{aligned}
& \frac{1}{2} \int_{0}^{1} \int_{0}^{h(t)+} \frac{\left[h^{-1}(y)\right]^{2}}{t^{2}} \nu(d y) d t \\
& \quad=\frac{1}{2} \int_{0}^{h(1)+} \int_{h^{-1}(y)}^{1} \frac{\left[h^{-1}(y)\right]^{2}}{t^{2}} d t \nu(d y) \\
& \quad=\frac{1}{2} \int_{0}^{h(1)+} h^{-1}(y)\left[1-h^{-1}(y)\right] \nu(d y)<\infty .
\end{aligned}
$$

Part 10. Part 3 of the proof shows that if (iii') is false, then a true statement is obtained by replacing 0 by 1 in the right hand side of (i'); and, thus, in the right hand side of (i). One could also use Blumenthal's $0-1$ law on page 57 of [3] to arrive at this conclusion. The proof is complete.

If one is "given" an increasing process with stationary independent increments-i.e. if one is given $\nu, F_{\nu}, g_{\nu}$, or $\varphi_{\nu}$-and if one is given $h \in M$, one might quite easily ascertain whether or not one of the statements (ii), (iii), or (iv) is true; thus one might easily conclude 
whether or not statements (i) and (i') are true. We now prove a similar theorem concerning the behavior of the sample paths for large values of $t$. The statement of the theorem is somewhat complicated by the fact that the behavior for large $t$ can depend on both the "small" and "large" jumps; whereas the behavior for small $t$ depends only on the "small" jumps.

THEOREM 2. Let $h \in M$. Let $\alpha \in(0, \infty)$ be defined by the equation $\alpha=\inf \{c: c t \geqq h(t)$ for $t \in[0, \infty)\}$. If $\alpha<\infty$, then the following statements are equivalent:

$$
\begin{aligned}
\text { ( i ) } \quad P\left\{X_{\nu}(t) \geqq h(t) \text { i.o. as } t \rightarrow \infty\right\}=0 ; \\
\quad\left(\mathrm{i}^{\prime}\right) \quad P\left\{X_{\nu}(t)>h(t) \text { i.o. as } t \rightarrow \infty\right\}=0 ; \\
\text { (aii) } \int_{0}^{\infty} x d_{2} F_{\nu}(t, x)<\alpha t \text { for one }(\text { all }) t \in(0, \infty) \text {; } \\
\text { (aiii) } \int_{0}^{\infty} y \nu(d y)=\int_{0}^{\infty} \nu[y, \infty) d y=\int_{0}^{\infty} \nu(y, \infty) d y<\alpha \text {; } \\
\text { (aiv) } g_{\nu}^{\prime}(0)<\alpha .
\end{aligned}
$$

If $\alpha=\infty$, then the following statements are equivalent:

( i ) $P\left\{X_{\nu}(t) \geqq h(t)\right.$ i.o. as $\left.t \rightarrow \infty\right\}=0$;

$$
\text { ( i' } \quad P\left\{X_{\nu}(t)>h(t) \text { i.o. as } t \rightarrow \infty\right\}=0 \text {; }
$$

(bii) $\int_{1}^{\infty}\left[1-F_{\nu}(t, h(t))\right] / t d t<\infty$;

$$
\text { (bii') } \int_{1}^{\infty}\left[1-F_{\nu}(t, h(t)+)\right] / t d t<\infty ;
$$

(biii) $\int_{1-}^{\infty} h^{-1}(y) \nu(d y)<\infty$;

$$
\text { (biii') } \int_{1}^{\infty} \nu[h(t), \infty) d t=\int_{1}^{\infty} \nu(h(t), \infty) d t<\infty \text {; }
$$

(biv) $\int_{1}^{\infty}\left[g_{\nu}\left(\frac{1}{h(t)}\right)-\frac{1}{h(t)} g_{\nu}^{\prime}\left(\frac{1}{h(t)}\right)\right] d t<\infty$.

If statements (i) and ( $\left.\mathrm{i}^{\prime}\right)$ are false, then true statements are obtained by replacing 0 by 1 in the right hand sides of these statements. If $\alpha=\infty$, then the truth or falsity of (i), (i'), (bii), (bii'), (biii), (biii'), and (biv) is not changed by replacing $h$ by $h_{c}$ or $H_{c}$. The integrals in (bii) and (bii') conceivably might not exist (even in the sense of $=\infty)$ : if so, either upper or lower integrals may be used.

REMARK. There exists $h \in M$ such that (i) is false. Also, there exists $h \in M$ such that (i) is true.

Proof. Part 0. We shall assume that the integrals in (bii) and (bii') exist (We do not exclude $=\infty$.): if not, only minor changes are needed in the proof.

Part 1. Obviously $(\mathrm{i}) \Rightarrow\left(\mathrm{i}^{\prime}\right)$ and $($ bii $) \Rightarrow\left(\right.$ bii' $\left.^{\prime}\right)$. 
Part 2. Note that $\int_{0}^{1-} y \nu(d y)<\infty$. Using this fact together with integration by parts (analogous to part 2 of the proof of Theorem 1), we easily deduce the two equalities in (aiii) and the equivalence of (biii) and (biii').

Part 3. We complete the proof in case $\alpha<\infty$. We have

$$
X_{\nu}(n t, \omega)=\sum_{k=1}^{n} X_{\nu}(k t, \omega)-X_{\nu}((k-1) t, \omega)
$$

The random variables $X_{\nu}(k t)-X_{\nu}((k-1) t), k=1, \cdots$ are mutually independent and identically distributed with distribution function $F_{\nu}(t, \cdot)$. Hence, by the law of large numbers,

$$
P\left\{\lim _{n \rightarrow \infty} \frac{X_{\nu}(n t)}{n}=\int_{0}^{\infty} x d_{2} F_{\nu}(t, x)\right\}=1 \text {. }
$$

By Theorem 4 of [5] we can, in fact, say that if

$$
\int_{0}^{\infty} x d_{2} F_{\nu}(t, x)<\infty
$$

then

$$
P\left\{X_{\nu}(n t)>n \int_{0}^{\infty} x d_{2} F_{\nu}(t, x) \text { i.o. as } n \rightarrow \infty\right\}=1 \text {. }
$$

Hence, using the fact that $h \in M$, the truth of the theorem for $\alpha<\infty$ will follow once it is shown that

$$
t \int_{0}^{\infty} y \nu(d y)=t g_{\nu}^{\prime}(0)=\int_{0}^{\infty} x d_{2} F_{\nu}(t, x)(\text { possibly }+\infty) .
$$

We have

$$
e^{-t g_{\nu}(u)}=\int_{0}^{\infty} e^{-u x} d_{2} F_{\nu}(t, x)
$$

For $u>0$, we can differentiate under the integral to obtain

$$
-t g_{\nu}^{\prime}(u) e^{-t g_{\nu}(u)}=-\int_{0}^{\infty} x e^{-u x} d_{2} F_{\nu}(t, x) . \quad \text { Now let } u \rightarrow 0
$$

and use the fact that $g_{\nu}$ is monotone. Hence, the right hand equality follows. We also have $g_{\nu}(u)=\int_{0}^{\infty}\left(1-e^{-u y}\right) \nu(d y)$. Differentiating and letting $y \rightarrow 0$, we obtain the left hand equality.

Part 4. We imitate part 3 of the proof Theorem 1 to show that $\left(\mathrm{i}^{\prime}\right) \Rightarrow\left(\right.$ biii' $\left.^{\prime}\right)$ and that, if (biii') is false a true statement is obtained by replacing 0 by 1 in the right hand side of $\left(i^{\prime}\right)$. Theorem 11.3 of [8], although not necessary, could be used here. 
Part 5. We assume that (biii') is true and $\alpha=\infty$ and we prove (i). Let $\beta>0$ be such that $\int_{\beta-}^{\infty} h^{-1}(y) \nu(d y)<1 / 2$. We define measures $\eta$ and $\xi$ on $(0, \infty)$ by the formulae

$$
\begin{aligned}
& \eta(B)=\nu(B \cap(0, \beta)) ; \\
& \xi(B)=\nu(B \cap \mid \beta, \infty)) .
\end{aligned}
$$

By what we proved in part 3 it follows that

$$
\begin{aligned}
& P\left\{X_{\eta}(t) \geqq h(t) / 2 \text { i.o. as } t \rightarrow \infty\right\} \\
& \quad \leqq P\left\{X_{\eta}(t) \geqq 2 t \int_{0}^{\beta-} y \eta(d y) \text { i.o. as } t \rightarrow \infty\right\}=0 .
\end{aligned}
$$

Hence, it will suffice to show that

$$
P\left\{X_{\xi}(t) \geqq h(t) / 2 \text { i.o. as } t \rightarrow \infty\right\}=0 \text {. }
$$

But this will follows if we can show that

$$
P\left\{X_{\hat{\xi} \circ(h / 2)}(t) \geqq t \text { i.o. as } t \rightarrow \infty\right\}=0 \text {; }
$$

for, as in part 4 of the proof of Theorem 1, we have

$$
X_{\xi \circ(h / 2)}(t, \omega) \geqq h^{-1}\left(2 X_{\xi}(t, \omega)\right) \text {. }
$$

We note that

$$
\begin{aligned}
& \int_{0}^{\infty} y(\xi \circ(h / 2))(d y)=\int_{0}^{\infty} h^{-1}(2 y) \xi(d y) \\
& \quad \leqq 2 \int_{0}^{\infty} h^{-1}(y) \xi(d y)=2 \int_{\beta-}^{\infty} h^{-1}(y) \nu(d y)<1 .
\end{aligned}
$$

The desired result follows by what was proved in part 3 .

The author would like to thank the referee for pointing out an error in the original calculations above. Also, he made several helpful suggestions about the organization of the paper.

Part 6. As in part 5 of the proof of Theorem 1, we can show that, if $\alpha=\infty$, then the truth or falsity of (i), (i'), (biii), and (biii') is unchanged if $h$ is replaced by $h_{c}$ or $H_{c}$.

Part 7. That (bii') $\Rightarrow$ (i) $\Rightarrow$ (bii) when $\alpha=\infty$ can easily be shown using methods analogous to those used in parts 6 and 7 of the proof of Theorem 1.

Part 8. Let $\alpha=\infty$. Then to show that (biii) $\Leftrightarrow$ (biv) we proceed in a manner similar to parts 8 and 9 of the proof of Theorem 1 . At one point the calculations become dissimilar: the appropriate calculation follows: 


$$
\begin{aligned}
& \frac{1}{2} \int_{1}^{\infty} \int_{0}^{h(t)+} \frac{y^{2}}{[h(t)]^{2}} \nu(d y) d t \\
& \quad=\frac{1}{2}\left[\int_{0}^{h(1)+} \int_{1}^{\infty}+\int_{h^{\prime}(1)+}^{\infty} \int_{h^{-1}(y)}^{\infty}\right] \frac{y^{2}}{[h(t)]^{2}} d t \nu(d y) .
\end{aligned}
$$

Now

$$
\left[\int_{0}^{h(1)+} y^{2} \nu(d y)\right]\left[\int_{1}^{\infty} \frac{1}{[h(t)]^{2}} d t\right]<\infty
$$

Since $h \in M$, we have

$$
\begin{aligned}
& \int_{h(1)+}^{\infty} \int_{h^{-1}(y)}^{\infty} \frac{y^{2}}{[h(t)]^{2}} d t \nu(d y) \leqq \int_{h(1)+}^{\infty} \int_{h^{-1}(y)}^{\infty} \frac{\left|h^{-1}(y)\right|^{2}}{t^{2}} d t \nu(d y) \\
& \quad=\int_{h(1)+}^{\infty} h^{-1}(y) \nu(d y)<\infty .
\end{aligned}
$$

The proof of Theorem 2 is complete.

We now prove a variation-type theorem.

Theorem 3. Let $h^{-1} \in M$ and $t \in(0, \infty)$. For almost all $\omega \in \Omega$ the following statements are true. If $\int_{0}^{1} h(y) \nu(d y)=\infty$, then, given $L<+\infty$, there exists $\delta(=\delta(t, \omega))>0$ such that

$$
\sum_{j=1}^{n} h\left(X_{\nu}\left(t_{j}\right)-X_{\nu}\left(t_{j-1}\right)\right)>L
$$

whenever $0 \leqq t_{j}-t_{j-1}<\delta$ and $t_{0}=0, t_{n}=t . \quad$ If $\int_{0}^{1} h(y) \nu(d y)<\infty$, then, given $\varepsilon>0$, there exists $\delta(=\delta(t, \omega))>0$ such that

$$
0 \leqq X_{\nu \circ h^{-1}}(t)-\sum_{j=1}^{n} h\left(X_{\nu}\left(t_{j}\right)-X_{\nu}\left(t_{j-1}\right)\right)<\varepsilon
$$

whenever $0 \leqq t_{j}-t_{j-1}<\delta$ and $t_{0}=0, t_{n}=t$.

Proof. Let $\mu=\nu \circ h^{-1}$. Because $h$ is concave downward it follows that $X_{\mu}(t)-\sum_{\jmath=1}^{n} h\left(X_{\nu}\left(t_{j}\right)-X_{\nu}\left(t_{j-1}\right)\right) \geqq 0$. If $\varepsilon>0$ is given then we can find $s_{1}, \cdots, s_{m}<t$ such that $X_{\mu}(t)-\sum_{i=1}^{m} h\left(J_{\nu}\left(s_{i}\right)\right)<\varepsilon$. We certainly can find $\delta\left[\right.$ even if $\left.\int_{0}^{1} h(y) \nu(d y)=\infty\right]$ such that

$$
\sum_{j=1}^{n} h\left(X_{\nu}\left(t_{j}\right)-X_{\nu}\left(t_{j-1}\right)\right) \geqq \sum_{i=1}^{n} h\left(J_{\nu}\left(s_{i}\right)\right)
$$

whenever $0 \leqq t_{j}-t_{j-1}<\delta$ and $t_{0}=0, t_{n}=t$. If $\int_{0}^{1} h(y) \nu(d y)=\infty$, we might still define $X_{\mu}(t)=\sum_{\tau \leqq t} h\left(J_{\nu}(\tau)\right)$, although $X_{\mu}(t)$ might conceivably equal $+\infty$. If $X_{\mu}(t)=+\infty$, then we can find $s_{1}, \cdots, s_{m}<t$ such that $\sum_{i=1}^{m} h\left(J_{\nu}\left(s_{i}\right)\right)>L$. Putting the statements of the above paragraph 
together we see that we are finished once we prove that $P\left\{X_{\mu}(t)=\right.$ $\infty\}=1$ assuming that $\int_{0}^{1} h(y) \nu(d y)=\infty$. We let $N_{j}(t, \omega)$ equal the number of $\tau \in[0, t]$ having the property that $J_{\nu}(\tau, \omega) \in\left[2^{-j-1}, 2^{-j}\right)$; $j=0,1, \cdots$. Then

$$
X_{\mu}(t) \geqq \sum_{j=0}^{\infty} h\left(2^{-j-1}\right) N_{j}(t) \geqq \frac{1}{2} \sum_{j=0}^{\infty} h\left(2^{-j}\right) N_{j}(t) .
$$

Hence,

$$
\begin{aligned}
E & \left\{\exp \left[-2 X_{\mu}(t)\right]\right\} \leqq E\left\{\exp \left[-\sum_{j=0}^{\infty} h\left(2^{-j}\right) N_{j}(t)\right\}\right. \\
& =\Pi_{j=0}^{\infty} E\left\{\exp \left[-h\left(2^{-j}\right) N_{j}(t)\right]\right\} \\
& =\Pi_{j=0}^{\infty} \exp \left\{-t \nu\left[2^{-j-1}, 2^{-j}\right)\left[1-\exp \left(-h\left(2^{-j}\right)\right)\right]\right\} \\
& =\exp \left\{-t \sum_{j=0}^{\infty}\left[1-\exp \left(-h\left(2^{-j}\right)\right)\right] \nu\left[2^{-j-1}, 2^{-j}\right)\right\} \\
& \leqq \exp \left\{-t \int_{0}^{1}\left[1-e^{-h(y)}\right] \nu(d y)\right\} \\
& \leqq \exp \left\{-t \text { (const.) } \int_{0}^{1} h(y) \nu(d y)\right\}=0 ;
\end{aligned}
$$

where we have used the fact that if $U$ is Poisson distributed with parameter $\eta$ and if $a$ is some nonnegative constant, then

$$
E\left\{e^{-a U}\right\}=\exp \left[-\eta\left(1-e^{-a}\right)\right] .
$$

We now prove an $L_{1}$ convergence theorem.

Theorem 4. Let $h^{-1} \in M$ and assume that $\int_{0}^{1} \nu[y, \infty) d h(y)<\infty$. Then

$$
\lim _{t \rightarrow 0} \int_{0}^{y}\left|\frac{1-F_{\nu}(t, x)}{t}-\nu[x, \infty)\right| d h(x)=0 .
$$

REMARK. By the central convergence criterion ([11], p. 311), we know that $\lim _{t \rightarrow 0}\left[1-F_{\nu}(t, y)\right] / t=\nu[y, \infty)$ for every continuity point $y$ of $\nu[\cdot, \infty)$.

Proof. Let $G(t, y)=F_{\nu}\left(t, h^{-1}(y)\right)$ and $\mu[y, \infty)=\nu\left[h^{-1}(y), \infty\right)$. Then Theorem 3 together with the central convergence criterion ([11], p. 311 ) gives us

$$
\begin{gathered}
\lim _{t \rightarrow 0} t^{-1} \int_{0}^{y-} h(x) d_{2} F_{\nu}(t, x)=\lim _{t \rightarrow 0} t^{-1} \int_{0}^{h(y)-} x d_{2} G(t, x) \\
=\int_{0}^{h(y)} x \mu(d x)=\int_{0}^{y} h(x) \nu(d x)
\end{gathered}
$$


for any $y$ which is a continuity point of $\nu[\cdot, \infty)$. Integration by parts together with the remark preceding this proof gives us

$$
\lim _{t \rightarrow 0} t^{-1} \int_{0}^{y}\left[1-F_{\nu}(t, x)\right] d h(x)=\int_{0}^{y} \nu[x, \infty) d h(x)
$$

The remark after the theorem together with the fact just proved implies the desired conclusion.

Except for the assertions involving $g(u)$, the four theorems generalize in the obvious way to the situation where $\nu$ is a measure on $R^{N}$ such that $\int_{0<|x|<1}|x| \nu(d x)<\infty$.

\section{BIBLIOGRAPHY}

1. Glen Baxter and J. M. Shapiro, On bounded infinitely divisible random variables, Sankjyā: The Indian Journal of Statistics, 22 (1960), 253-260.

2. D. Bierens de Hahn, Nouvelles Tables d'Intégrales Définies, G. E. Stechert and Company, 1939 (corrected the edition of 1867).

3. R. M. Blumenthal, An extended Markov property, Trans. Amer. Math. Soc. 85 (1957), 52-72.

4. R. M. Blumenthal and R. K. Getoor, Sample function of stochastic processes with independent increments, J. Math. Mech. 10 (1961), 493-516.

5. K. L. Chung and W. H. J. Fuchs, On the distribution of values of sums of random variables, Number 6, Memoirs of the Amer. Math. Soc., 1951.

6. J. L. Doob, Stochastic Processes, John Wiley and Sons, Inc., London, 1953.

7. Bert Fristedt, Generalized variations, continuity, acd Hausdorff measures of sample functions of stochastic processes with independent increments, Thesis, MIT, 1963.

8. Edwin Hewitt and Leonard J. Savage, Symmetric measures on cartesian products, Trans. Amer. Math. Soc. 80 (1955), 470-501.

9. A. Khinchin, Sur la croissance locale des processus stochastiques homogènes à accroissements indépendants (Russian article and French resume), Akad. Nauk. SSSR, Izvestia, Ser. Math. 3 (1939), 487-508.

10. —, Zwei Sätze ïber stochastische Prozesse mit stabilen Verteilungen (Russian article and German resume), Mathematicheskii Sbornik 3 (1938), New Series, 577-584. 11. Michel Loève, Probability Theory, 2nd edition, D. Van Nostrand Company, Inc., Princeton, New Jersey, 1960.

Received November 18, 1965. This research was supported in part by the Air Force Office of Scientific Research under grant Af-AFOSR 62-252. Theorem 3 together with some of the easier parts of the proof of Theorem 1 are contained in the author's Ph. D. thesis completed at MIT under the direction of Henry P. McKean, Jr.

Carleton College, Northfield, Minnesota

AND

University of Minnesota 



\section{PACIFIC JOURNAL OF MATHEMATICS}

\section{EDITORS}

H. SAMELSON

Stanford University

Stanford, California

J. P. JANS

University of Washington

Seattle, Washington 98105
J. DugundJI

University of Southern California

Los Angeles, California 90007

RICHARD ARENS

University of California

Los Angeles, California 90024

\section{ASSOCIATE EDITORS}

E. F. BECKENBACH

B. H. NeumanN

F WolF

K. YOSIDA

\section{SUPPORTING INSTITUTIONS}

UNIVERSITY OF BRITISH COLUMBIA

CALIFORNIA INSTITUTE OF TECHNOLOGY

UNIVERSITY OF CALIFORNIA

MONTANA STATE UNIVERSITY

UNIVERSITY OF NEVADA

NEW MEXICO STATE UNIVERSITY

OREGON STATE UNIVERSITY

UNIVERSITY OF OREGON

OSAKA UNIVERSITY

UNIVERSITY OF SOUTHERN CALIFORNIA
STANFORD UNIVERSITY

UNIVERSITY OF TOKYO

UNIVERSITY OF UTAH

WASHINGTON STATE UNIVERSITY

UNIVERSITY OF WASHINGTON

AMERICAN MATHEMATICAL SOCIETY CHEVRON RESEARCH CORPORATION TRW SYSTEMS

NAVAL ORDNANCE TEST STATION

Printed in Japan by International Academic Printing Co., Ltd., Tokyo Japan 


\section{Pacific Journal of Mathematics}

\section{Vol. 21, No. $1 \quad$ November, 1967}

Friedrich-Wilhelm Bauer, Der Hurewicz-Satz................... 1

D. W. Dubois, A note on David Harrison's theory of preprimes . ......... 15

Bert E. Fristedt, Sample function behavior of increasing processes with stationary, independent increments ..................... 21

Minoru Hasegawa, On the convergence of resolvents of operators....... 35

Søren Glud Johansen, The descriptive approach to the derivative of a set function with respect to a $\sigma$-lattice ....................... 49

John Frank Charles Kingman, Completely random measures ............ 59

Tilla Weinstein, Surfaces harmonically immersed in $E^{3} \ldots \ldots \ldots \ldots \ldots . . \ldots 9$

Hikosaburo Komatsu, Fractional powers of operators. II. Interpolation spaces ......................................... 89

Edward Milton Landesman, Hilbert-space methods in elliptic partial differential equations ...................................... 113

O. Carruth McGehee, Certain isomorphisms between quotients of a group algebra ........................................ 133

DeWayne Stanley Nymann, Dedekind groups .................. 153

Sidney Charles Port, Hitting times for transient stable processes ......... 161

Ralph Tyrrell Rockafellar, Duality and stability in extremum problems involving convex functions . ............................ 167

Philip C. Tonne, Power-series and Hausdorff matrices . . .............. 189 